\title{
Axing Wnt signals
}

\author{
Felipe De Sousa E Meloํㅡㄹ Jan Paul Medema ${ }^{1}$ \\ ${ }^{1}$ Laboratory of Experimental Oncology and Radiobiology, Center for Experimental Molecular Medicine, Academic Medical Cen- \\ ter, University of Amsterdam, 1105AZ Amsterdam, The Netherlands \\ Cell Research (2012) 22:9-11. doi:10.1038/cr.2011.141; published online 30 August 2011
}

Wnt signaling is a highly conserved and context-dependent signal transduction pathway that belongs to the so-called morphogens. Wnt signals are instrumental during development, but also play a central role in tissue homeostasis. For instance, in the intestine Wnt signaling is crucial for both stem cell maintenance and proliferation in the transient amplifying compartment [1]. This Wnt-dependent stem cell regulatory role is observed in diverse adult tissues, but is not a general feature as Wnt signals can also drive differentiation of cells, such as mesenchymal stem cells (MSCs) [2]. Wnt signaling can be divided into a canonical and a noncanonical pathway. The non-canonical pathway mainly mediates migration, while the canonical signals are mostly conveyed by $\beta$-catenin, a transcription factor that is actively repressed in the cytoplasm in the absence of Wnt signals. $\beta$-catenin levels are kept under control by a multi-protein complex composed of the tumor suppressor adenomatous polyposis coli (APC); two kinases, casein kinase 1 (CK1) and glycogen synthase kinase 3 beta (GSK3- $\beta$ ); and Axin2 that serves as a scaffold to hold the complex together. This complex allows CK1 and GSK3- $\beta$ to phosphorylate $\beta$-catenin at specific serine and threonine residues, priming its recognition

Correspondence: Jan Paul Medema

E-mail: j.p.medema@amc.nl by the U3 ubiquitin ligase $\beta$-transducin repeat-containing protein $(\beta-\mathrm{TRCP})$. Consequently, $\beta$-catenin is ubiquitinated and targeted for proteosomal degradation (Figure 1A). However, in the presence of extracellular Wnt ligands, $\beta$-catenin degradation is halted. Wnt binds the membrane-bound receptor complex formed by frizzled (Fzd) and low-density lipoprotein receptor-related protein 5/6 (LRP5/6). As a result, the destruction complex is dissolved by a still poorly understood mechanism. This allows $\beta$-catenin to accumulate in the cytosol and, subsequently, translocate into the nucleus. There, it associates with the lymphoid enhancer factor/Tcell factor (LEF/TCF) family of transcription factors, converting them from repressors to activators of transcription. These steps are followed by additional nuclear events that ultimately trigger a complex transcriptional program depending on the cellular context that will direct cell fate, cell proliferation, and stem cell maintenance or cellular differentiation (Figure 1B) (See review by MacDonald et al., 2009) [3].

It is clear that a tight control of the pathway is necessary to ensure homeostasis in adult tissues. The consequences of the Wnt pathway are best studied in the gastrointestinal tract where Wnt activity maintains the stem cell compartment. Constitutive activation of the Wnt pathway initiates the transition of normal intestinal epithelium towards metaplasia [4], while inhibition of the signal leads to attrition of the epithelial layer [5]. Given its widespread impact in both tissue maintenance and disease, Wnt pathway inhibitors are actively pursued $[6,7]$. Several screening programs using large chemical compound libraries to modulate the Wnt signal transduction route in both a positive and negative fashion have emerged.

In a recent paper published in Cell Research, Gwak et al. screened for small molecules that could potentiate the Wnt signaling cascade [8]. Using a cell-based assay with a large synthetic chemical compound library, they identified SKL2001 as a potent activator of $\beta$-catenin transcriptional activity. Previously, several stimulating compounds have been shown to activate the Wnt signal transduction pathway. These mainly target the kinase activity of GSK3- $\beta$ [9] and as such prevent the adequate phosporylation of $\beta$-catenin at Ser33/37/Thr41 residues which would normally lead to its proteosomal degradation. Gwak et al. found that SKL2001 treatment also results in decreased phosphorylation at these residues, but found no evidence that the kinase activity of GSK3- $\beta$ was affected. In contrast, SKL2001 altered the integrity of the destruction complex. Using immunoprecipitation assays, Gwak et al. demonstrated that SKL2001 competes with GSK3- $\beta$ and precludes the interaction between $\beta$-catenin and the Axin2 scaffolding protein, a model that is further supported by molecular modeling. Preventing the interaction of $\beta$-catenin with Axin2 impinges on the 


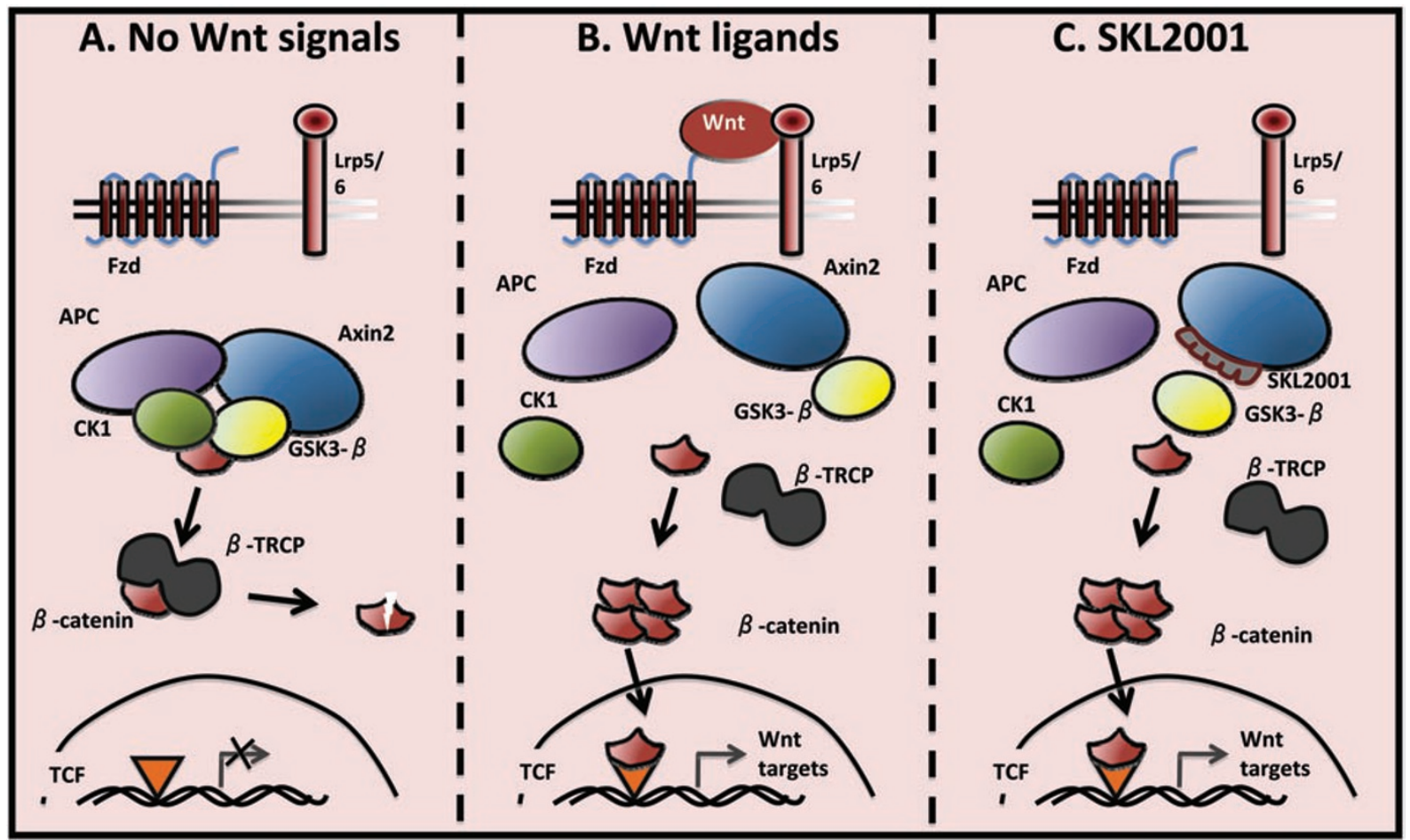

Figure 1 (A) In the absence of Wnt ligands, $\beta$-catenin is kept under low cytosolic level by the destruction complex. This complex contains Axin2 and APC, which present $\beta$-catenin to the 2 kinases CK1 and GSK3- $\beta$, enabling its phosphorylation at specific serine and threonine residues. This primes $\beta$-catenin recognition by $\beta$-TRCP, which targets it for proteosomal degradation. In the nucleus, TCF transcription factors are repressed and Wnt target gene transcription is inhibited. (B) When Wnt ligands bind to Fzd and LRP5/6 co-receptors, the destruction complex is dissolved. $\beta$-catenin is stabilized, can accumulate in the cytosol, and subsequently, translocate into the nucleus where it converts TCF into a transcriptional activator. These events trigger an efficient transcription of genes that are important regulators of stem cell fate, cell proliferation as well as cell fate determination. (C) The small molecule agonist SKL2001 binds to Axin2 and precludes its interaction with GSK3- $\beta$, leading to an inefficient targeting of $\beta$-catenin for degradation. Therefore, $\beta$-catenin can accumulate and form active transcription factor complexes with TCF proteins in the nucleus, even in the absence of external signal.

degradation of $\beta$-catenin and thereby allows translocation of $\beta$-catenin into the nucleus, switching on the Wnt program (Figure 1C). Importantly, SKL2001 can be competed out by overexpression of Axin2, confirming the model. Gwak et $a l$. therefore unravel an effective and more specific way to artificially drive Wnt signals since it does not affect the activity of kinases, which may play alternative roles. Although the potential toxicity and side effects of SKL2001 have yet to be evaluated in mouse models, its physiological potency was further assessed on a model of MSC differentiation. SKL2001 will activate the Wnt pathway in MSCs and as such can readily skew differentiation towards the osteoblastic lineage at the expense of adipocyte differentiation, in line with previous reports $[2,10]$. These in vitro observations need further physiological support in relevant models where total body fat and bone mass can be critically evaluated. Nevertheless, the potency and selectivity of this compound are of clear interest and should be evaluated further.

Other adult tissues that critically depend on Wnt signals would predictably benefit from such small molecule agonist. As mentioned above, Wnt signaling is intimately linked with the biology of the intestinal epithelium where it maintains and drives the stem cells. Especially in case of epithelial damage that occurs due to inflammation or directly by toxic substances, a great demand rests on stem cells to maintain the tissue integrity. Natural Wnt agonists, such as members of the $\mathrm{R}$-spondin family, have recently been shown to protect intestinal stem cells in mice after radiation-induced intestinal damage. More specifically it reduces epithelial cell death and improves functional regeneration of the intestinal tract [11]. This highlights the great promises held by natural and chemical compounds activating the Wnt pathway in regenerative medicine. Similarly, such compounds would strongly influence the in vitro growth of intestinal organoid cultures. Although their use 
in regenerative medicine may seem far down the road, compounds that can be produced under GMP standards will clearly facilitate this development.

Of course there is also a dark side to such Wnt activating compounds. Virtually all colorectal cancer (CRC) patients initiate cancer lesions upon aberrant activation of the Wnt pathway and several other tumors display aberrant activation as well. It is therefore to be anticipated that forced activation of Wnt signals might lead to the initiation of hyperplasia. In vivo activation of Wnt with small drugs will therefore likely walk a thin line between achieving the beneficial effects whilst avoiding the unwanted ones.

\section{References}

1 Medema JP, Vermeulen L. Microenvironmental regulation of stem cells in intestinal homeostasis and cancer. $\mathrm{Na}$ ture 2011; 474:318-326.

2 Day TF, Guo X, Garrett-Beal L, Yang Y. Wnt/beta-catenin signaling in mesenchymal progenitors controls osteoblast and chondrocyte differentiation during vertebrate skeletogenesis. Dev Cell 2005; 8:739-750.

3 MacDonald BT, Tamai K, He X. Wnt/ beta-catenin signaling: components, mechanisms, and diseases. Dev Cell 2009; 17:9-26.

4 Kinzler KW, Vogelstein B. Lessons from hereditary colorectal cancer. Cell 1996; 87:159-170.

5 Korinek V, Barker N, Moerer P, et al. Depletion of epithelial stem-cell compartments in the small intestine of mice lacking Tcf-4. Nat Genet 1998; 19:379383.

6 Chen B, Dodge ME, Tang W, et al. Small molecule-mediated disruption of
Wnt-dependent signaling in tissue regeneration and cancer. Nat Chem Biol 2009; 5:100-107.

7 de Sousa EM, Vermeulen L, Richel D, Medema JP. Targeting Wnt signaling in colon cancer stem cells. Clin Cancer Res 2011; 17:647-653.

8 Gwak J, Hwang SG, Park HS, et al. Small molecule-based disruption of the Axin $/ \beta$-catenin protein complex regulates mesenchymal stem cell differentiation. Cell Res 2012; 22:237-247.

9 Ying QL, Wray J, Nichols J, et al. The ground state of embryonic stem cell self-renewal. Nature 2008; 453:519523.

10 Ross SE, Hemati N, Longo KA, et al. Inhibition of adipogenesis by Wnt signaling. Science 2000; 289: 950-953.

11 Bhanja P, Saha S, Kabarriti R, et al. Protective role of R-spondin1, an intestinal stem cell growth factor, against radiation-induced gastrointestinal syndrome in mice. PLoS One 2009; 4:e8014. 